\title{
Mujeres lideresas constructoras de paz en la comuna 1 de Medellín: una aproximación a las paces cotidianas construidas desde abajo*
}

\author{
[Versión en Castellano]
}

Peace-building Women Leaders in Medellin's Comuna 1: A Grassroots Approach to Establishing Daily Peace

Mulheres líderes construtoras de paz no setor 1 de Medellin: uma aproximação aos passos diários construídos a partir de abaixo

Recibido el 23 de junio, 2020. Aceptado el 30 de agosto, 2020.

\author{
Juan-Pablo Acosta-Navas** \\ https://orcid.org/0000-0003-1350-0795
}

Colombia

Para citar este artículo:

Acosta-Navas, Juan-Pablo (2021).

Mujeres lideresas constructoras

de paz en la comuna 1 de

Medellín: una aproximación a

las paces cotidianas construidas desde abajo.

Ánfora, 28(50). 75-102.

https://doi.org/10.30854/anfv28.n50.2021.712

Universidad Autónoma de

Manizales. ISSN 0121-6538.

E-ISSN 2248-6941.

CC BY-NC-SA 4.0

\section{Resumen}

Objetivos: analizar las contribuciones de las mujeres lideresas constructoras de paz de la Comuna 1 de Medellín en el ejercicio de su liderazgo comunitario, que aporta a la consolidación de un concepto de construcción de paz desde abajo y desde una perspectiva crítica y relacional de los derechos humanos. Metodología: investigación de carácter socio jurídico con enfoque cualitativo, desarrollada a partir de un estudio de caso con las mujeres lideresas constructoras de paz de la Comuna 1 de Medellín. En la ejecución de la investigación se combinaron técnicas

\footnotetext{
* Este artículo constituye uno de los productos de la investigación concluida con la cual se optó al título de Magíster en Derecho de la Universidad de Antioquia, modalidad investigativa. El autor declara que no hubo conflictos de intereses en la ejecución del proyecto de investigación.

** Magíster en Derecho. Abogado. Profesor de la Universidad de Antioquia. Miembro de los grupos de investigación: Derecho y Sociedad; y Conflictos, Violencias y Seguridad Humana. Correo electrónico: juan.acostan@udea.edu.co
} 
documentales (análisis de conceptos y de literatura especializada), conversacionales (entrevistas semiestructuradas) e interactivas (trabajo de campo en territorio). Resultados: se encontraron manifestaciones de las paces cotidianas a nivel territorial por parte de las mujeres lideresas, además de diversas expresiones de resistencia y reexistencia encaminadas a resignificar la dignidad humana y la acción colectiva a través de apuestas ético-políticas en la construcción de paces en la Comuna 1. Conclusiones: las mujeres lideresas constructoras de paz en el territorio estudiado van un paso adelante de la teoría hegemónica de los derechos humanos y de los postulados de la paz liberal, su comprensión e interpretación de la sociedad y sus conflictos resulta de avanzada; esto ejemplifica muy bien cómo se puede continuar luchando en el ámbito sociopolítico por una sociedad más justa y en paz con una perspectiva desde abajo, para materializar esas paces cotidianas en sus contextos barriales y comunitarios.

Palabras-clave: Construcción de paz desde abajo; Mujeres lideresas constructoras de paz; Paces cotidianas; Paz femenina; Investigación sobre la paz; Consolidación de la paz.

\section{Abstract}

Objective: to analyze the contributions of peace-building women leaders from Medellín's Comuna 1 in the exercise of their community leadership, which contributes to the consolidation of a grassroots peace-building concept and a critical and relational perspective of human rights. Methodology: research of a socio-legal nature with a qualitative approach, developed from a case study with peace-building women leaders of Medellín's Comuna 1. In the execution of the research, documentary (analysis of concepts and specialized literature), conversational (semi-structured interviews) and interactive techniques (field work in the territory) were combined. Results: regular, i.e. daily evidence of peace at the territorial level by women leaders were found, in addition to various expressions of resistance and re-existence aimed at reaffirming human dignity and collective action through ethical-political stakes in building peace in Comuna 1. Conclusions: women leaders who build peace in the studied territory are one step ahead of the hegemonic theory of human rights and the postulates of liberal peace, their understanding and interpretation of society and its conflicts is advanced. This exemplifies very well how one can continue to fight in the sociopolitical sphere for a more just and peaceful society from a grassroots perspective, to materialize continued peace in their neighborhood and community contexts.

Keywords: Grassroots peace-building; Peace-building women leaders; Everyday peace; Female peace; Research on peace; Consolidation of peace. 


\section{Resumo}

Objetivo: para analisar as contribuições das mulheres líderes construtoras de paz do Setor 1 de Medellín no exercício de sua liderança comunitária, o que contribui para a consolidação de um conceito de construção da paz desde abaixo e de uma perspectiva crítica e relacional dos direitos humanos. Metodologia: pesquisa de natureza sóciojurídica com abordagem qualitativa, desenvolvida a partir de um estudo de caso com as principais mulheres construtoras da paz do Setor 1 de Medellín. Técnicas documentais (análise de conceitos e literatura especializada), conversacional (entrevistas semiestrusas) e interativa (trabalho de campo em território) foram combinadas na execução da pesquisa. Resultados: se encontraram manifestações de ritmo diário no nível territorial por mulheres líderes, bem como várias expressões de resistência e re-existência destinadas a ressignificar a dignidade humana e a ação coletiva através de apostas ético-políticas na construção da paz no setor 1. Conclusões: as mulheres líderes construtoras de paz no território estudado estão um passo à frente da teoria hegemônica dos direitos humanos e dos postulados da paz liberal, sua compreensão e interpretação da sociedade e seus conflitos estão avançados; isso exemplifica muito bem como podemos continuar a lutar na esfera sociopolítica por uma sociedade mais justa e pacífica com uma perspectiva desde abaixo, para materializar esses passos diários em seus contextos de bairro e comunitário.

Palavras-chave: Construção de paz desde abaixo; Mulheres líderes construtoras de paz; Passos diários; Paz feminina; Pesquisa da Paz; Construção da paz. 


\section{Introducción}

Este artículo derivado de la investigación de Maestría puso su foco sobre aquello que se denomina paz; un concepto ambivalente alrededor del cual también pueden construirse discursos para justificar las violencias y los conflictos, por paradójico que parezca.

La paz tiene múltiples manifestaciones, dimensiones, expresiones y campos de estudio: educación y pedagogía (para la paz), cultura y construcción (de paz), la paz como derecho y como deber, los estudios de paz, el pacifismo, el antimilitarismo y la no violencia son algunos ejemplos, pero todo lo mencionado sugiere una necesidad humana fundamental: poner en el centro de las discusiones académicas, políticas y de sociedad, eso que denominamos paz.

La historia de las últimas décadas en Colombia tiene como una de sus caras más crudas a la violencia en sus múltiples manifestaciones, o mejor, a las violencias en plural en los términos de Galtung (2016), quien propone tres tipologías: la violencia directa, la violencia estructural y la violencia cultural. Posteriormente, se abordarán estos conceptos. En el contexto colombiano resulta imperativo preguntarse por qué la paz parece tan esquiva cuando se constituye en el anhelo y el clamor de muchos.

\section{Metodología}

La metodología desarrollada en esta investigación es de carácter socio jurídico y se ubica en el método cualitativo, que debe entenderse más allá de un simple conjunto de técnicas de recolección de información. En este sentido, Galeano-Marín (2011) afirma que es un modo de encarar el mundo a partir de los sujetos sociales entre sí y de las relaciones de estos con sus contextos. Por eso, se considera que el método cualitativo aborda las realidades subjetivas e intersubjetivas, se centra en los actores, busca comprender desde diversas perspectivas y sujetos, se basa en lo cotidiano para la comprensión de esa realidad y hace énfasis en lo local, lo micro, lo regional (Galeano-Marín, 2011, pp. 16-24). Y esa mirada micro y regional (o territorial en este caso) fue el derrotero para el trabajo de campo.

Así, este texto se articuló a partir reflexiones teóricas sobre el concepto de derechos humanos (condensadas en otros productos derivados de la investigación en mención) de la mano con un estudio de caso con las Mujeres Lideresas Constructoras de Paz (MLCP) de la Comuna 1 de Medellín. En la ejecución de la investigación se combinaron técnicas documentales (análisis de conceptos, 
de instrumentos normativos y de literatura especializada), conversacionales (entrevistas semiestructuradas) e interactivas (trabajo de campo en el territorio).

En cuanto al enfoque en la investigación jurídica, Villabella (2015) menciona que esta puede clasificarse en teórica, empírica y mixta (p. 926). En ese orden de ideas, esta investigación fue realizada desde un enfoque mixto, porque combinó un componente teórico robusto con la dimensión empírica derivada de la interacción con las mujeres en el territorio para, posteriormente, contrastar dicha propuesta teórica de las paces cotidianas con la forma en la que esta se relaciona, se tensiona y se vivencia por la comunidad.

Ahora, en general, este artículo desarrolla, en un primer momento, una contextualización sucinta sobre el conflicto armado en Colombia y en Medellín. Luego, aborda el concepto de Mujer Lideresa Constructora de Paz (MLCP) y su relación con la paz femenina. Posteriormente, articula las reflexiones teóricas con el trabajo de campo desarrollado en la Comuna 1 de Medellín con las MLCP. Para tal propósito, se analiza la paz más allá de la mera ausencia de guerra, la paz desde lo simbólico y la paz desde la organización comunitaria. Por último, presenta un acápite de consideraciones finales, que más que arrojar conclusiones, pretende generar preguntas y posibles líneas de investigación a futuro.

\section{Resultados}

Los principales hallazgos se condensan en las siguientes ideas: en primer lugar, es necesario desjuridizar y desestatalizar los derechos humanos ${ }^{1}$ en la medida en que estos se hacen y deshacen todos los días como productos socioculturales que son, pese a la influencia jurídico-normativa, sobre la cual reposan.

En segundo término, la reconceptualización de la construcción de paz en clave crítica de derechos humanos debe asumir una doble dimensión, ya que la acción colectiva y la capacidad de agencia de las mujeres lideresas constructoras de paz no son suficientes en sí mismas, por muy loables que sean. Por tanto, sus propuestas y experiencias particulares deberían influir e impactar en los diseños institucionales de políticas públicas, que permitan por ejemplo, la plena garantía,

\footnotetext{
1. Otro de los productos de la investigación aquí referida se ocupa de manera particular de la revisión de instrumentos del Derecho Internacional de los Derechos Humanos desde una perspectiva sociocrítica. El artículo será publicado en próximos días bajo el título: "Los derechos humanos en disputa: una lectura crítica en clave relacional".
} 
específicamente, de derechos de las mujeres lideresas y de las poblaciones vulnerables y victimizadas en general.

Y en tercer lugar, la construcción de paces cotidianas en clave crítica de derechos humanos conjugando los elementos anteriores, permitirá hacer un viraje hacia una visión relacional (Sánchez-Rubio, 2018a), en la cual no importen tanto los instrumentos normativos que consagran los derechos humanos o sentencian la paz como derecho y deber, sino que tenga como foco a los sujetos que construyen esas relaciones humanas día tras día.

\section{Contexto y generalidades del conflicto armado en Colombia y en Medellin}

\section{Sobre la guerra que desangra a una sociedad afligida.}

Según el Centro Nacional de Memoria Histórica, CNMH, (2018), en Colombia entre 1958 y septiembre de 2018 la confrontación armada dejó 261.619 víctimas fatales, de las cuales 214.584 fueron víctimas civiles y 46.675 personas que participaban directamente en las hostilidades ${ }^{2}$ (CNMH, 2018, p. 1). Lo anterior, señala que solo el $17,8 \%$ de las víctimas fatales habían tomado parte en las hostilidades en el conflicto armado, mientras que el $82 \%$ de las víctimas fueron personas civiles que no participaban directamente en este. De acuerdo con las líneas precedentes, es un hecho que en la confrontación bélica en Colombia más de 8 de cada 10 víctimas mortales han sido personas civiles.

Apostarle a la paz desde todos los escenarios posibles, sea en el ámbito familiar, en el relacionamiento social o, incluso, de manera más amplia apoyando procesos organizativos y comunitarios, articulándose con movimientos políticos que defiendan dicha bandera o sumando esfuerzos en el contexto académico para construir una apuesta intelectual ligada a una agenda investigativa. Estos son pasos necesarios para consolidar la construcción de paz en el país y deben operar bajo la sinergia y no como proyectos éticos y políticos aislados entre sí.

2. El Centro Nacional de Memoria Histórica, CNMH, (2018), los denomina “combatientes", sin embargo, en estricto rigor jurídico, la categoría combatiente, de acuerdo con el DIH, solo es aplicable a los Conflictos Armados Internacionales (CAI), por lo cual se optó por utilizar el término de participación directa en las hostilidades dispuesto en el Artículo 3 Común a los Cuatro Convenios de Ginebra de 1949 y en su Protocolo Adicional II de 1977, relativos a los Conflictos Armados No Internacionales (CANI). 


\section{Sobre la paz en el posacuerdo y un conflicto armado que persiste.}

Recuerda Restrepo (2016) que la paz en Colombia no es una victoria militar de ningún partido o gobierno, sino simplemente el reconocimiento del derecho humano ya conquistado que le pertenece a todos los habitantes del país para vivir tranquilos y con dignidad; así pues, "La paz no es un concepto que se acomoda con un decreto, o la firma del acuerdo, ni se resuelve con votar el sî" (Restrepo, 2016, p. 59). Dicho de otro modo, la paz no puede ser impuesta por una norma, pues es evidente que su consolidación implica una mayor y sustancial complejidad.

Por esa razón los colectivos de mujeres, de jóvenes, de víctimas, de desplazados, de poblaciones étnicas y, por supuesto, la comunidad académica, deben apostarle al afianzamiento de esa paz tan anhelada, ya que, como afirma Lederach (2008), "Las dificultades para alcanzar una paz duradera en procesos de violencia prolongada indican que sabemos algo más sobre cómo poner fin a algo doloroso y perjudicial para todo el mundo, pero sabemos bastante menos sobre cómo construir algo deseado" (p. 80).

Pese al horizonte de paz derivado de la firma del Acuerdo Final, las trabas a la consolidación de una paz completa, con justicia social y equidad para los colombianos hace pensar que tal propósito será una tarea de largo aliento. Desde 2016 y hasta agosto de 2020 se contabilizan más de 224 homicidios en contra de firmantes de la paz, otrora miembros de las extintas FARC-EP (registros que son sistematizados por el mismo Partido Político).

En tanto, desde la firma del Acuerdo Final hasta el 21 agosto de 2020 ya se registran 1.000 lideresas y líderes sociales asesinados (Instituto de Estudios para el Desarrollo y la Paz [Indepaz], 2020). Líderes y lideresas como aquellos que participaron en esta investigación, hombres y mujeres cuyas vidas extinguen las balas de los actores armados, mientras ellos y ellas ponen todos sus esfuerzos por la construcción de un país mejor, en paz, y por defender las causas de sus familias, sus comunidades y sus territorios. Vidas segadas por la violencia de manera paradójica, por pedir a gritos paz.

\section{Paz femenina y Mujeres Lideresas Constructoras de Paz (MLCP).}

Antes de desarrollar estos dos conceptos fundamentales vale la pena destacar que la Organización de Naciones Unidas (2000) en cabeza de su Consejo de Seguridad ${ }^{3}$ emitió la Resolución 1325 del 2000, aprobada en la sesión 4213

\footnotetext{
3. El Consejo de Seguridad de las Naciones Unidas es uno de los seis órganos principales de la Organización, y tiene como propósitos fundamentales garantizar la paz y la seguridad internacional, los cuales a su vez se constituyen en los pilares de la Carta de San Francisco de 1945, instrumento mediante el cual se constituye
} 
del 31 de octubre del mismo año. Dicha Resolución relativa a "Mujeres, Paz y Seguridad" reconoce que las mujeres y las niñas son sujetos de especial afectación en el marco de los conflictos armados, pero a su vez insta a los Estados miembros de la ONU (de la cual Colombia es parte) a adoptar decisiones y mecanismos institucionales que prevengan la agresión y las violencias basadas en género, así como permitir la participación de mujeres y niñas en la solución de conflictos y los procesos de paz que se deriven de ellos (Organización de Naciones Unidas, 2000, pp. 2-3).

$\mathrm{El}$ anterior, es un antecedente internacional relevante en la medida en que las Resoluciones del Consejo de Seguridad se consideran obligatorias para los Estados pertenecientes a la ONU. Sin embargo, en el marco de la negociación con las extintas FARC-EP y el gobierno nacional, los plenipotenciarios nombrados por la Presidencia de la República fueron todos hombres; en ese sentido, el gobierno de turno negaba que las mujeres habían experimentado el conflicto armado en su vida diaria siendo sus cuerpos un territorio en el cual una parte de ese conflicto se había desarrollado (Fernández-Matos, 2019, p. 116).

Ante esta exclusión deliberada de las mujeres en la negociación, en el Encuentro Nacional de Mujeres por la Paz dado en Bogotá en el 2012, las mujeres allí reunidas publicaron el 4 de diciembre de dicho año un Manifiesto denominado: La paz sin las mujeres ¡no va! (Mujeres por la Paz, 2012), el Manifiesto abría con la siguiente frase:

En estos dos días de debates, encuentros y de compartir experiencias, reafirmamos nuestro compromiso ético y político con la construcción de la paz y la salida política al conflicto social y armado; nos declaramos insumisas frente al patriarcado y el capitalismo y nos negamos a continuar siendo las pactadas de la cultura patriarcal, queremos ser pactantes del nuevo contrato social que deriva del proceso de diálogo (Mujeres por la Paz, 2012, párr. 2).

Y más adelante amplían la discusión sobre el contenido del derecho humano a la paz en un contexto relacional y no exclusivamente jurídico, pues señalan que no basta silenciar los fusiles o pacificar las zonas de conflicto armado, sino que "La paz significa desmilitarizar los territorios, las mentes y la palabra" (Mujeres por la Paz, 2012), lo que fue sentenciado por las mujeres en este Manifiesto:

La paz no es el silenciamiento de los fusiles en lo público y en lo privado. La paz implica hacer realidad la justicia social para todos y todas sin distinción de etnia,

la Organización. En las últimas décadas ha cobrado relevancia la discusión en el área del Derecho Internacional sobre el carácter vinculante de las Resoluciones que emite este Órgano, lo cual se considera un hecho si se tienen en cuenta los artículos 39-45 de la Carta de San Francisco. 
sexo, religión, postura política o condición económica; significa garantizar los derechos a la verdad, la justicia y la reparación de todas las víctimas; erradicar la violencia como ejercicio de la política y la negación del otro y la otra como práctica cotidiana (Mujeres por la Paz, 2012, párr. 5).

Ahora bien, en los talleres realizados en el marco de esta investigación se evidenció que la participación de las mujeres en los escenarios locales y barriales de construcción de paz es mayor a la de los hombres. Tal afirmación se hace a partir de la experiencia particular en el trabajo de campo y en las interacciones comunitarias que soportaron este proyecto (esto será ampliado en las líneas siguientes).

Ese nivel de participación de las mujeres en los espacios comunitarios relacionados con la paz se constituyó en uno de los hallazgos tempranos de la investigación, lo cual guarda coherencia con las experiencias mencionadas en líneas precedentes en la medida en que durante negociaciones del Acuerdo Final y al quedar en evidencia la exclusión deliberada que se había hecho respecto al rol de las mujeres en dichos espacios, esto precipitó sus demandas por tener mayores posibilidades de real participación e impulsó su interés en los años venideros (2012 - actualidad) por tomar la vocería en los distintos procesos de construcción de paces cotidianas y territoriales que de algún modo tienen eco y aportan al proyecto de construcción de paz a escala nacional.

\section{Las mujeres lideresas como referentes teóricas en sí mismas}

Con base en lo señalado, las mujeres lideresas se erigieron en el centro de la investigación y, en esa medida, se hizo una apuesta ética, política, académica, epistemológica y metodológica, por considerar dicha participación de las mujeres no como un elemento accidental dentro de la investigación, sino como las referentes teóricas en sí mismas del proyecto. Las mujeres, desde sus lugares de enunciación y desde sus saberes populares, comunitarios y ancestrales acumulados, ofrecen una lección a la academia acerca de la validez, vigencia y trascendencia de sus reflexiones, las cuales han sido elaboradas a partir del rol que desempeñan en sus comunidades y bien podrían suponer una ruptura o un desbalance con las formulaciones sobre paces que se erigen desde las universidades y los centros de investigación por la distancia evidente entre la teoría de los estudios de paz y áreas afines con respecto a lo que ocurre en los territorios.

Aquellas mujeres lideresas son una voz autorizada en sus barrios por tomar la iniciativa y liderar los procesos de reivindicación de derechos ante distintos actores institucionales y no institucionales; además, por replicar las experiencias exitosas de sus procesos de formación y capacitación como lideresas con las 
demás mujeres y miembros de la comunidad, fungiendo como unas verdaderas multiplicadoras del conocimiento popular y articuladoras de los procesos organizativos.

Para ejemplificar lo anterior, la Mujer Lideresa (en adelante ML) 2 señalaba:

"La academia tiene su discurso para los académicos, yo he sido muy resistente a las universidades que traen su discurso, acá maneje el discurso del territorio, que lo que usted me esté diciendo sea la realidad de lo que está pasando en el territorio (...) En el papel de las mujeres, [tiene] mucha importancia la participación, porque una golondrina no hace verano. Si las mujeres nos unimos, dejamos ese egoísmo y nos unimos a construir paz, [es] a través del diálogo y la concertación en el territorio” (Entrevista a Mujer Lideresa 2, 2019).

Las mujeres lideresas que habitan conviven, coexisten y resisten y reexisten en la Comuna 1, fueron las protagonistas de esta investigación que procuró visibilizar cómo ellas desde lo colectivo le apuestan a la construcción de escenarios de paces cotidianas. Y pese a que la investigación al desarrollarse en el marco de un programa de Maestría siguió una lógica académica convencional con respecto la formulación del proyecto, su ejecución pretendió situar a las mujeres en el centro no con fines instrumentales ni exclusivamente metodológicos, sino como un reconocimiento de su ejercicio y su rol como protagonistas políticas cruciales para los procesos de construcción de paces que comienzan a afianzarse en la ciudad.

Para concluir este apartado, es pertinente aproximarse al concepto de reexistencia que será trabajado en líneas posteriores y que ha sido desarrollado, entre otros, por los profesores Adolfo Albán y José Rosero (2016), quienes entienden el concepto como una expresión de dignidad en sí misma, como la forma comunal de recrear la vida, la cual

Ha de darnos las bases para que lo culturalmente sustentable sea la concepción de la vida misma en condiciones de dignidad, no negociables con el capital, sino que definitivamente la concepción del mundo no se corresponda con el beneficio a ultranza de la naturaleza y se constituya en un posibilitador de garantía de larga duración de todas las especies vivas, incluido el ser humano (Albán y Rosero, 2016, p. 39).

De allí que la existencia, resistencia y re-existencia de las mujeres lideresas constructoras de paz participantes en esta investigación sea una apuesta política no solo por la paz, sino también por una vida en condiciones dignas. 


\section{La paz femenina}

Las autoras Díez y Mirón (2004) mencionan que la forma de nominar y denominar a las personas, a las cosas y a las situaciones puede entrañar distintos prejuicios y estereotipos. En ese sentido, el estudio de la categoría de mujeres da cuenta de un vasto campo semántico que empieza incluso por la denominación en singular (mujer) y en plural (mujeres); en el primer caso, supone hablar de un prototipo ideal o de una idea general y abstracta, mientras que al emplear mujeres se pretende destacar la singularidad de ellas como sujetos individuales, las autoras también mencionan la clásica asociación entre mujeres y paz y entre hombres y guerra, lo cual permite pensar la relación antiquísima entre guerra y paz, pero sin caer en reduccionismos o determinismos biológicos:

Tradicionalmente, la Paz se ha asociado y exigido a las mujeres. El porqué de la asociación de las mujeres con la Paz lo encontramos en la idea de la abundancia y fertilidad que se va a mantener como una constante a lo largo de la historia, tanto para la Paz como para las mujeres. La Paz es fértil, genera abundancia, del mismo modo que el papel que se ha destacado de las mujeres se ha centrado en su fertilidad y en ser generadora de vida (Díez y Mirón, 2004, p. 72).

Coincide con lo anterior Inés Sánchez-Díaz (2017) quien revisa y recupera las investigaciones de las autoras citadas con anterioridad e incorpora otras voces femeninas a sus reflexiones; así, Sánchez-Díaz (2017) afirma que

La paz, por lo tanto, no es un ámbito específico del género femenino, ni las mujeres poseen una predisposición natural para la misma, sino que su construcción es una tarea que concierne a ambos sexos por igual. Sin embargo, es innegable el hecho de que las movilizaciones de mujeres han incluido muy a menudo la paz entre sus reivindicaciones, tal y como puso de manifiesto la alianza entre el sufragismo y el pacifismo primero, y las uniones recurrentes entre feminismo y pacifismo después (p. 269).

Existe algo muy interesante que emerge en la lectura conjunta y armónica de las autoras o investigadoras que podríamos denominar, académicas con respecto a las autoras o investigadoras populares y comunitarias; es decir, las mujeres lideresas constructoras de paz y participantes centrales de esta investigación.

Esa emergencia señala una relación y casi una correspondencia entre las preocupaciones o reflexiones de las investigadoras académicas y las de las mujeres lideresas o populares de la Comuna 1 de Medellín, las agendas de paz femenina -o paz feminista- parecen no variar sustancialmente, pues las demandas y banderas 
de inclusión, de equidad, de oportunidades y, en un sentido más amplio, que las mujeres sean escuchadas y sus voces resuenen y sean tenidas en cuenta a la hora de la toma de decisiones de interés público, es una coincidencia que no resulta accidental. Esa correspondencia o diálogo entre las investigadoras académicas y las mujeres feministas activistas y lideresas señala una particular sintonía entre las agendas de las mujeres a pesar de las diferencias entre el campo académico y de activismo comunitario.

La zona nororiental, en la cual se ubica la Comuna 1, representa un territorio particular de Medellín por los altos niveles de violencia que se vivieron en determinadas épocas; así pues, estas mujeres lideresas ejercen sus roles en contextos altamente conflictivos. Para ilustrar esto, en la figura 1 se relacionan los asesinatos selectivos asociados al conflicto armado, los cuales se concentraron principalmente en tres comunas: la Comuna 1: Popular; la Comuna 13: San Javier; y la Comuna 3: Manrique, registrando estas 3 comunas entre 900 y 1.600 homicidios entre 1980 y 2014 (Centro Nacional de Memoria Histórica [CNMH], et al., 2017), cabe resaltar que tanto la Comuna 1 (Popular) como la Comuna 3 (Manrique) hacen parte de la zona nororiental en la división administrativa de Medellín.

Figura 1. Asesinatos selectivos por Comunas de Medellín, 1980-2014

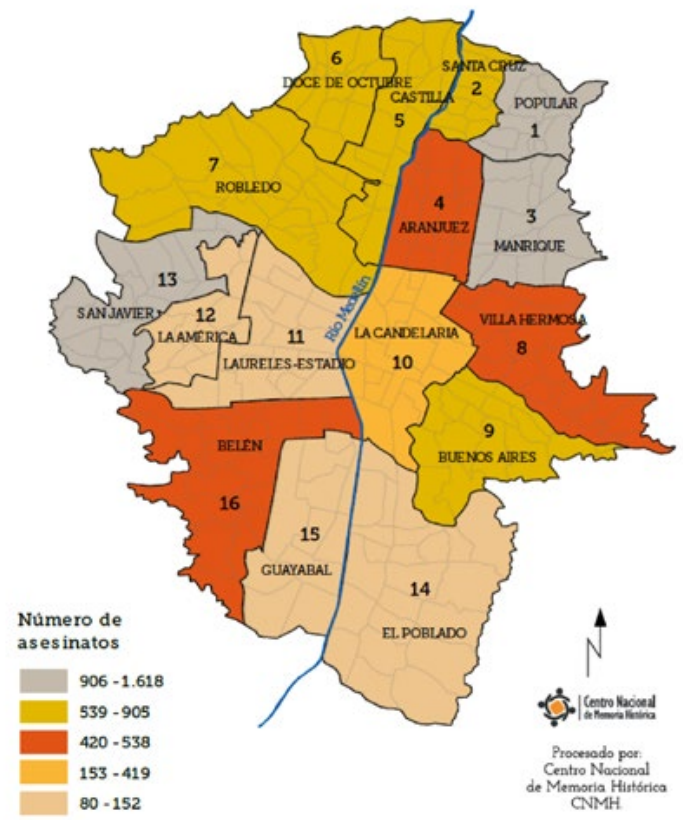

Fuente: $\mathrm{CNMH}$, et al. ( 2017) 
Retomando la discusión, la referencia a las autoras académicas citadas con anterioridad conecta de manera precisa con las reflexiones de la Mujer Lideresa 2, quien comentó que "Somos las mujeres quienes lleva[mos] la iniciativa en estos procesos [de paz], es nuestro instinto maternal, nosotras tenemos más instinto de defender la vida, y no solo la mía, sino también la de las personas que están alrededor" (Entrevista a Mujer Lideresa 2, 2019, p. 2).

Desde luego, las mujeres lideresas pueden ver con cier to escepticismo el papel de la academia en general, y en particular de las investigadoras o investigadores que se interesan por sus procesos comunitarios dentro de la investigación. En la misma entrevista, ML2 señala que:

"La academia tiene su discurso para los académicos, yo he sido muy resistente a las universidades que traen su discurso, acá maneje el discurso del territorio, que lo que usted me esté diciendo sea la realidad de lo que está pasando en el territorio" (Entrevista a Mujer Lideresa 2, 2019, p. 3).

Más allá de esa suerte de desconfianza o escepticismo -sumamente legítimo por la instrumentalización de las comunidades por parte de investigadoras e investigadores durante décadas- ML2 acepta que a veces el comportamiento de las mismas mujeres conlleva a divisiones o tensiones dentro de los espacios comunitarios en los cuales participan y, por lo tanto, aboga por un movimiento de mujeres más unido en Medellín para lograr construir paz en los territorios, una paz que tenga sello femenino y que responda a las necesidades particulares de las mujeres en realidades convulsas y complejas como la de Medellín:

"En el papel de las mujeres, [tiene] mucha importancia la participación, porque una golondrina no hace verano. Si las mujeres nos unimos, dejamos ese egoísmo y nos unimos a construir paz, [es] a través del diálogo y la concertación en el territorio" (Entrevista a Mujer Lideresa 2, 2019, p. 3).

La ML10 también reconoce el papel crucial de las mujeres como agentes y constructoras de paz teniendo en cuenta que antes de construir paz, es necesario analizar el conflicto y buscar su transformación:

"Las mujeres siempre nos hemos destacado en masa para la resolución de los conflictos, pero aún nos falta mucha preparación. Es muy positivo que las mujeres nos estemos movilizando y saliendo de la casa al escenario de lo público [pero] el asunto del empoderamiento requiere un tiempo" (Entrevista a Mujer lideresa 10, 2019, p. 5). 
Antes que revivir un determinismo biológico que reduzca la ecuación a mujeres igual a paz y hombres igual a guerra, lo que se debe fortalecer es la "feminización de la paz", lo cual va en una doble vía: primero, la sociedad debe interiorizar las prácticas tradicionales y actitudes pacíficas femeninas incorporando a la mujer en ese ejercicio de construcción activa de la paz. Así lo afirma Ivonne Wilches en su investigación para UNIFEM y el PNUD denominada: "Paz con género femenino":

La inclusión de las mujeres en los procesos de paz apunta directamente a un requisito de justicia, a un derecho femenino y a la posibilidad de que la igualdad que demanda el enfoque de género apoye un proceso de construcción de democracia social (Wilches, 2010, p. 91).

Segundo, debe entenderse que la paz estructural -donde hombres y mujeres participen en grados similares de libertad e igualdad para construir una verdadera cultura de paz-solo es posible si existe una paz de género; en suma, debe transformarse el binomio tradicional hombres-guerra y mujeres-paz por una cultura de paz en la que participen todas y todos (Díez y Mirón, 2004, pp. 88-90). El concepto de paz femenina da paso a la aproximación a la categoría de Mujer Lideresa Constructora de Paz (MLCP).

\section{Mujeres Lideresas Constructoras de Paz (MLCP)}

En el análisis documental se evidenció que el concepto de Mujer Lideresa Constructora de Paz (MLCP) no aparece de manera expresa en la literatura consultada, aunque existen otras fórmulas cercanas como mujer constructora de paz. Sin embargo, en el trabajo de campo y en las interacciones comunitarias emerge el concepto de MLCP de forma implícita en el diálogo con las mujeres pues su agenda feminista, comunitaria y política se enmarca en su liderazgo para construir la paz territorial. En esa medida, las mujeres participantes de esta investigación no solo contribuyen a la construcción de paz o paces cotidianas $\mathrm{y}$ territoriales, sino que también reivindican su rol de mujeres lideresas como articuladoras de esos procesos de paces.

Incluso, conceptos como construcción de paz (como acción) han sido abordados por la literatura de manera profusa en tanto verbo o acción (construir paz), pero no se identifica el mismo volumen de información frente al sujeto que la construye; dicho de otro modo, ha sido una preocupación en el mundo académico durante las últimas décadas preguntarse: ¿cómo se construye la paz?, 
pero a la pregunta ¿quién es el sujeto que construye esa paz? le falta un mayor desarrollo.

Hoy las mujeres son artífices de múltiples procesos organizativos y comunitarios de incidencia y de construcción de paces; pero no debe perderse de vista que su corporalidad ha sido históricamente un territorio en disputa y un botín de guerra. En ese orden de ideas, la feminista argentina Rita Segato (2013) se refiere a la corporalidad de la mujer como zona de disputa y como estrategia bélica en sí misma, recordando las atrocidades ocurridas en los conflictos armados de Ruanda (1994) y Yugoslavia (1992-1995) donde aparece la violencia sexual ya no como mero daño incidental, sino como un arma cuyo daño letal es simultáneamente material y moral: "La impresión que emerge de ese nuevo accionar bélico es que la agresión, la dominación y la rapiña sexual ya no son, como fueron anteriormente, complementos de la guerra, daños colaterales, sino que han adquirido centralidad en la estrategia bélica" (Segato, 2013, p. 19).

Anabel Garrido, por su parte, expresa cómo incluso después de la firma de un cese al fuego o de un acuerdo de paz, pensando en el contexto colombiano, es posible que la violencia -o las violencias en plural- perduren en contra de las mujeres, así lo expresa al afirmar:

El territorio se encuentra como disputa del conflicto por los actores armados, donde las mujeres se muestran como parte del territorio de conquista, que es poseído, en ambos casos, por hombres. Esta violencia es uno de los elementos claves en el conflicto colombiano, y muestra dinámicas que pueden permanecer después de los acuerdos de paz (Garrido, 2019, p. 119).

En este punto resulta pertinente aproximarse a la categoría de lideresa social, apelando al profesor de la Universidad de Antioquia James Granada, quien señala que "la definición de líder [o lideresa] social la da el mismo activismo y no tanto los liderazgos que se ejercen dentro de una institución” (Granada, 2019, p. 9). Esto se evidencia con claridad en la presente investigación, pues las mujeres lideresas participantes tienen un acumulado empírico dado por el territorio y no por la burocracia o la participación exclusiva en espacios institucionales. El profesor Granada complementa su definición de líder o lideresa social arguyendo que:

Es toda aquella persona que ejerce un liderazgo en el ámbito de una organización, de una comunidad o de movimientos de diversa índole: sociales, políticos y hasta religiosos. Esto se encuentra mucho en los barrios y veredas, donde el trabajo de base los convierte en líderes. A veces se trata de activistas muy visibles, 
que ejercen su liderazgo alejados de ciertas centralidades y por eso están más expuestos a las amenazas y a los ataques (Granada, 2019, p. 9).

Se cierra este acápite proponiendo pues una formulación propia a ese concepto de mujer lideresa constructora de paz, la cual se constituye como aquella mujer que en el ejercicio de su liderazgo barrial o comunitario trabaja por regenerar el tejido social y sanar las secuelas físicas y psicológicas derivadas del conflicto armado para dejar de ser nombrada exclusivamente como víctima de esa confrontación y transformar esa condición en acciones encaminadas a convertirse en una parte activa en la búsqueda y consolidación de la paz, construyéndola en las distintas dimensiones del territorio.

\section{El rol de las mujeres lideresas constructoras de paz}

\section{Pensar los derechos humanos y la construcción de paz más allá del registro liberal.}

Para pensar y re-pensar la construcción de paz como se propuso en la investigación de la cual se deriva este artículo, resulta imprescindible superar el paradigma del liberalismo en el cual los derechos humanos o la paz son posibles solo en la medida en que se privilegie la propiedad privada y prevalezca el libre mercado. Ese sujeto destinatario de derechos en la tradición liberal moderna eurocéntrica ha de superarse para lograr aproximaciones otras a las luchas por la dignidad, que por la herencia occidental recibida en países occidentalizados como Colombia, se expresan bajo la fórmula (o el traje) de los derechos humanos, con base en ello, resulta pertinente pensar los derechos humanos y la paz más allá de esa receta del liberalismo como lo aconseja la jurista Ratna Kapur (2018) en su más reciente obra: Gender, Alterity and Human Rights: Freedom in a Fishbowl; la profesora Swethaa Ballakrishnen escribe en su reseña del texto:

If meaningful freedom is what we are after, she counsels, we need to look past the language of human rights towards the transformative potential of other, nonliberal registers. Extending her own metaphor, it is not just about changing the water or the container, reward lies beyond the fishbowl altogether (Ballakrishnen, 2019, p. 2).

En efecto, pensar más allá del registro liberal posibilita las luchas por la dignidad, incluso en lenguajes distintos al de los derechos humanos o la paz liberal, pues estos conceptos aunque potentes, representan el proceso histórico de occidente y las victorias conseguidas por las élites masculinas eurocentradas, 
así pues, más que cambiar el recipiente (la pecera de los derechos humanos y la paz), debemos ir más allá de la pecera para alcanzar la dignidad, máxime, en contextos de sociedades no industrializadas y empobrecidas por los procesos de dominación colonial del pasado como la colombiana, y tratándose de las mujeres populares subalternizadas de esas sociedades.

En este caso concreto, se trata de mujeres lideresas que a diario deben lidiar con la estigmatización, la persecución y las múltiples formas de violencias (directa, estructural y simbólica), que el ejercicio de su liderazgo causa en aquellos sectores políticos y armados que viven por y para la guerra, y que tan pronto sus intereses se ven comprometidos, recurren a la violencia directa generando amenazas, intimidaciones, desplazamientos e incluso, son capaces de extinguir la vida de aquellas personas cuyo propósito no es otro que aportar a la construcción de paz en el país.

Esa mujer lideresa construye la paz en las distintas dimensiones del territorio: la dimensión espacial en su barrio, su comuna y su ciudad, la dimensión relacional que evoca la forma en que esa mujer lideresa interactúa con conocidos y desconocidos, vecinos y núcleo familiar, en suma, en su relación con los demás, y, por último, la dimensión corporal y personal, entendiendo que el cuerpo femenino otrora castigado y violentado por la guerra, se constituye en el primer territorio en el cual la mujer lideresa ha de construir paz y así poder reproducirla y multiplicarla en la dimensión relacional y espacial.

Max Yuri Gil (citado por Rendón, 2019) coordinador de la macrorregión Antioquia y Eje Cafetero de la Comisión para el Esclarecimiento de la Verdad, la Convivencia y la No Repetición, menciona: "Antioquia es una zona donde la guerra pasó y sigue pasando, sigue siendo el departamento con mayor número de líderes asesinados, con graves problemas de amenazas y ejecuciones extrajudiciales" (Rendón, 2019, p. 1).

En efecto, el departamento de Antioquia y su capital Medellín, han sido epicentro de algunas de las manifestaciones más degradantes, aberrantes y deshumanizadoras del conflicto armado, el conflicto pasó, sigue pasando y seguirá pasando mientras la paz no se convierta en el punto prioritario en las agendas de las instituciones pues las mujeres lideresas constructoras de paz, al igual que otros movimientos sociales y grupos poblacionales -generalmente aquellos más vulnerables, subalternos o que han padecido de manera directa el conflicto- no pueden nadar a contracorriente para generar escenarios de paces posibles mientras que las administraciones locales y el gobierno nacional sigan dando la espalda a la paz, no solo con una actitud pasiva u omisiva, pues también existen claras manifestaciones de hostilidad que impiden alcanzar esa anhelada paz completa. 


\section{La paz: más allá de la ausencia de guerra.}

Con todo lo anterior, parece emerger algo que la literatura quizá no haya abordado con suficiencia. Mientras que la paz negativa se ha asociado y estudiado desde la perspectiva de la ausencia de guerra, el silencio de los fusiles y la superación de las muertes violentas asociadas a la violencia directa; la paz positiva comprende de manera holística todas las necesidades básicas que deben ser satisfechas para lograr paz, pues sumado al cese de la confrontación armada, esa paz positiva también requerirá de alcanzar, en los términos de Galtung (2016) explorados con anterioridad, una paz estructural en la cual ningún ser humano sea explotado y cuente con la garantía de las condiciones materiales de existencia y a su vez, se precisará de una paz cultural en la cual la violencia simbólica no sea ejercida por ningún miembro de la sociedad estableciendo jerarquías de género, clase, nacionalidad o raza.

Este complejo entramado teórico y práctico de la relación entre paz directa (como paz negativa) sumada con la paz positiva (como paz estructural y paz cultural) deja al desnudo que las necesidades básicas insatisfechas son un primer factor que no permite alcanzar una paz completa a esta sociedad.

Superar la violencia directa y lograr que las personas no mueran más como consecuencia de los disparos, sin duda constituiría un gran logro para la sociedad colombiana, sin embargo, recuperando algo dicho por ML2, “¿de qué sirve hablar de paz cuando se tiene el estómago vacío?”. La paz, en ese orden de ideas comienza por algo más simple y probablemente más fácil de erradicar que la violencia directa en sí misma.

Garantizar que ninguna colombiana y ningún colombiano sienta hambre supone un primer peldaño para la superación de las violencias y la consecución de una paz verdadera, completa e integral, eso es justo lo que menciona entre líneas ML2 (ML2, Diario de Campo: abril 29, 2019, p. 17): "La paz se siente desde el estómago, desde nuestro vientre”.

\section{La paz desde lo simbólico.}

La figura 2 muestra unas manillas elaboradas con mostacillas, que tienen tejida la palabra PAZ con diversos colores. Las manillas fueron entregadas a las mujeres lideresas participantes de la investigación en forma de agradecimiento y reconocimiento a su labor de liderazgo, y también, como una manera de sellar nuestro compromiso entre academia y comunidad por defender y construir paz territorial, para lo cual, como se ha mencionado anteriormente, resulta crucial el papel activo de las mujeres a tan laboriosa empresa. 
Figura 2. Las manillas de la Paz

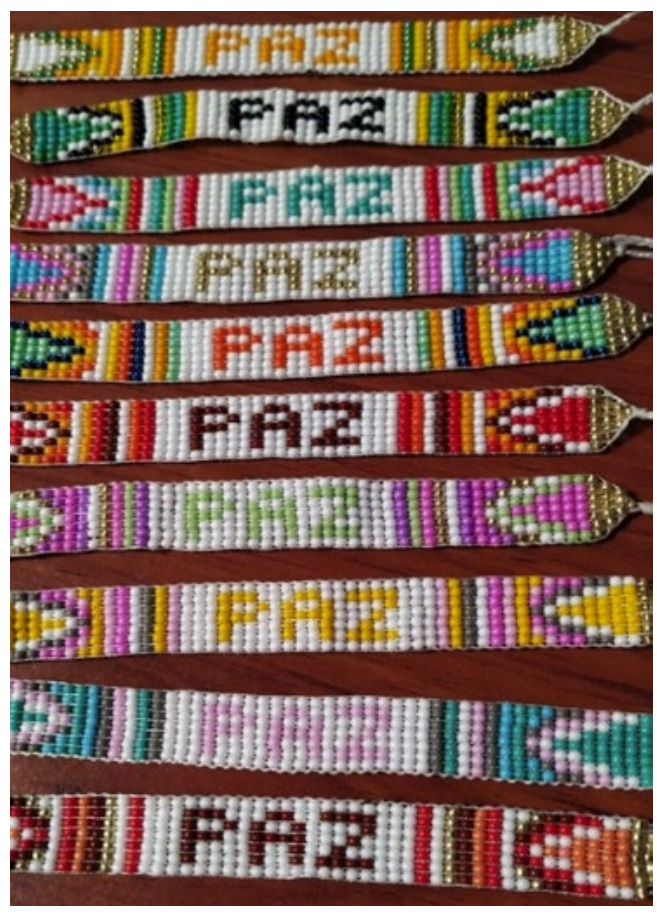

Fuente: elaboración propia

Así, es posible evidenciar cómo las mujeres de la Comuna 1 relacionan sus experiencias cotidianas y sus historias de vida para dotar de contenido eso que denominamos paz. Es claro que la comprensión de la paz de estas mujeres lideresas supera con creces el concepto limitado de paz negativa entendida como ausencia de guerra, y una conceptualización profunda de paz positiva se refleja en sus dichos y sentires en tanto integran en dicha reflexión otros elementos, que desde el punto de vista jurídico son a su vez derechos (y en un sentido más amplio, derechos fundamentales y humanos), como el caso de la educación o la alimentación, esto guarda relación también como el ejercicio de liderazgo de las mujeres en sus territorios.

\section{La paz desde la organización comunitaria y su impulso por las mujeres lideresas.}

La paz no es solo un concepto abstracto o teórico pese a la abundante literatura existente. La paz se vive, se hace y se rehace a diario por parte de 
los actores sociales, la paz requiere y precisa de las mujeres, pero también se necesita abandonar la idea de masculinidad asociada al guerrero pues resulta un absurdo mantener esa visión. Por tal motivo, tanto hombres como mujeres en una sociedad, como la nuestra, en pleno posacuerdo deben apoyar de manera decidida los procesos de construcción de paces en el nivel territorial, de ciudad y nacional. Parafraseando una expresión difundida por colectivos feministas a lo largo y ancho del mundo, la paz será feminista o no será.

Los derechos humanos y la paz, más allá de su dimensión jurídico-normativa en tanto derechos, tienen un alto contenido político que no se agota en el mero reconocimiento formal de su existencia. Por el contrario, su permanente reconstrucción y reformulación en el escenario de lo político es lo que los dota de valía, pues deben ser los actores sociales, representantes de esa idea abstracta de sociedad civil los que pueden incidir en la construcción de una idea de paz no estatalista, localizada y que se nutra de las permanentes fluctuaciones que como sociedad tenemos.

Por lo anterior, la paz no es un hecho ni un derecho dado, su contenido está en constante pugna y, por esa razón, los actores sociales, desde sus experiencias vitales, sus luchas cotidianas y sus victorias en el ámbito sociopolítico pueden contribuir enormemente a desjuridizar el derecho a la paz para comenzar a reconocer que en tanto derecho, es la sociedad civil la que debe y puede establecer sus alcances con el objetivo de construir escenarios de paz en un país desangrado por la guerra.

El rol de las MLCP demuestra que estas mujeres tienen una vocación ética y política por la construcción de escenarios de paz territorial en sus barrios y comunas; sin embargo, esta paz por la cual abogan se caracteriza por ser mucho más compleja que la mera ecuación del macro conflicto: ausencia de conflicto armado = paz. Esta concepción de paz desde las mujeres lideresas entiende que existen prioridades para existir y coexistir, habitar y cohabitar sus territorios para re-existir; silenciar los fusiles es necesario, pero no suficiente.

Parafraseando de nuevo a ML2 ¿cómo puedo hablar de paz si tengo hambre? Esta expresión simple, cotidiana y a la vez desgarradora, exhibe las desigualdades manifiestas de una sociedad como la colombiana. En efecto, con hambre no puede haber paz y menos en un escenario como el de algunos barrios periféricos de Medellín en el cual la violencia armada no cesa. Esas manifestaciones de la micro conflictividad pasan inadvertidas en los grandes medios de comunicación en los que importa más quién muere a causa de la violencia directa, que la supervivencia de aquellos que la resisten y abogan por una paz completa.

Ahora, puede que las armas se depongan, las balas se destruyan y los violentos se reincorporen a la vida en la comunidad política, pero mientras no estén garantizadas las condiciones materiales de existencia para el grueso de la 
sociedad que hoy padece las nefastas consecuencias del capitalismo crudo que gobierna al país como soberano incuestionable, esa paz no será más que ficticia.

Las manillas de mostacilla elaboradas con la palabra paz en el centro es una expresión simple y a la vez profunda, sobre cómo la paz va más allá de un acuerdo entre las partes enfrentadas, de la persecución a los grupos armados o de la captura y desarticulación de bandas y pandillas en la ciudad. La paz que se anhela parte de un supuesto concreto: la paz comienza por nosotros mismos y esa manilla es una expresión simbólica de cómo las mujeres lideresas al sortear todas las adversidades cotidianas que esta sociedad les impone, se encuentran trabajando desde el ser y el hacer para sus familias, comunidades y territorios por la construcción de paces, por la construcción de otro mundo posible y mejor.

\section{Conclusiones}

Como se anunció, la investigación de la cual se deriva este artículo buscó tejer una relación entre el acumulado del conocimiento académico sobre la construcción de paz, con el saber popular y situado de las mujeres lideresas constructoras de paz que desde su lugar de enunciación particular como articuladoras de los territorios en los cuales habitan o ejercen su liderazgo, trabajan por y promueven una sociedad en paz. Los dichos, saberes, expresiones y análisis de las mujeres lideresas expresados a lo largo de los talleres, interacciones comunitarias, conversaciones espontáneas y entrevistas, arrojan en la práctica, que estas mujeres lideresas son tenidas en cuenta dentro de la investigación como referentes teóricas en sí mismas.

Asimismo, debe desligarse la idea de que la paz solo existe en la medida en la que la garantice el Estado, pues si bien este juega un rol fundamental para poder crear escenarios de paz, es tan solo uno de los actores que debe sumar a la ecuación pues la construcción de paz está en cabeza en mayor medida de sujetas y sujetos, cuyas experiencias particulares y comunitarias son las que pueden generar senderos para caminar la paz y consolidarla en los territorios.

Tanto en el derecho interno como en el derecho internacional, las pretensiones en cuanto a la garantía y la consolidación de la paz y de los derechos humanos son vastas; sin embargo, traducir esas consagraciones o demandas de justicia social, de equidad y de respeto por la vida solo serán posibles si la sociedad toma conciencia de su papel como actor político para materializar ese catálogo de derechos, puesto que: 
La creación de normas [nacionales o internacionales] no soluciona los problemas estructurales de una sociedad que le teme a la diferencia y que repudia las otredades; por esta razón, los procesos de transformación política, institucional y ciudadana se han tornado lentos para solucionar los obstáculos jurídicos y culturales que supone la materialización de derechos de los grupos poblacionales vulnerables. Si bien [la expedición de normas jurídicas] es una herramienta relevante para alcanzar estas reivindicaciones, no resulta suficiente en tanto no ocurran procesos de movilización que permitan convertir las consagraciones de normas abstractas en transformaciones [políticas] de realidades particulares (Acosta-Navas, 2019, p. 57).

La relación entre los derechos humanos en clave crítica, la paz como derecho humano y la construcción de paces desde abajo impulsada por los movimientos sociales y, en este caso, por las mujeres lideresas de la Comuna 1 participantes de esta investigación, se hace evidente tras lo expuesto. Una teoría hegemónica de los derechos humanos que parta del provincialismo eurocéntrico para justificar la existencia, los elementos o las condiciones de consolidación de la paz (liberal) deja por fuera otras epistemologías, comprensiones y construcciones conceptuales de lo que la paz significa.

Wolfgang Dietrich menciona de manera categórica que "La paz no se puede producir o exportar, la paz que no se relaciona con lugares concretos nunca tendrá poder social alguno y permanecerá como una abstracción en las mentes de los investigadores para la paz” (Dietrich, 2006, p. 454).

Las mujeres lideresas de Medellín que participaron en esta investigación son la mejor evidencia de la necesidad de una relectura de la paz en clave de derechos humanos, pero fuera del recetario liberal y de la matriz colonial en la cual se incrusta el origen histórico del concepto occidental de derechos humanos. Las mujeres lideresas de la zona nororiental de Medellín construyen paz desde lo que puede denominarse como la microfísica de la emancipación (Sánchez-Rubio, $2018 b)$.

En esa medida, son acciones pequeñas en difusión, pero gigantescas en alcance y en la apuesta política que representan. La paz no se construye con la firma de documentos o ratificación de instrumentos internacionales; la paz se construye caminando los territorios, mediando en los conflictos comunitarios, respetando y escuchando al otro y a la otra, trabajando la empatía y generando confianza en las comunidades, pues recordando las palabras de Eduardo Galeano (2015): "Mucha gente pequeña, en lugares pequeños, haciendo cosas pequeñas, puede cambiar el mundo” (p. 1). 
Las mujeres lideresas constructoras de paz en la Comuna 1 van un paso adelante de la teoría hegemónica de los derechos humanos y de la paz liberal. Ellas, de manera consciente o inconsciente ponen en práctica los postulados de la teoría crítica al ubicar en el centro de la discusión al ser humano antes que a las normas jurídicas que dicen proteger a esos sujetos. La academia, como la élite social que representa, tiene aún mucho por aprender de sujetos políticos subalternos como estas mujeres lideresas; probablemente el ejercicio de su liderazgo comunitario no esté cimentado de manera fehaciente en un listado interminable de autores, de filosofías y teorías políticas, de corrientes críticas o de literatura jurídica especializada, pero su comprensión e interpretación de la sociedad avanzada y ejemplifica muy bien cómo se puede continuar luchando en el ámbito sociopolítico por una sociedad más justa y en paz.

La reconceptualización de la construcción de paz en clave crítica de derechos humanos se sintetiza en los siguientes elementos: en primer lugar, desjuridizar y desestatalizar los derechos humanos, entendiendo que estos emergen, se consolidan y se rehacen día a día y en pequeña escala, las comunidades heterogéneas a lo largo y ancho del planeta establecen sus agendas de lucha por sociedades más justas en los términos en los que sus contextos particulares sociopolíticos, religiosos o económicos lo demanda. Ello implica pensar en una agenda de derechos humanos más allá de la fórmula estatal y del ordenamiento jurídico internacional en cabeza de esa ficción denominada "comunidad internacional" y de las Organizaciones Internacionales como la ONU en el ámbito global, o la OEA en el ámbito regional (y los respectivos órganos de ambas).

En segundo término, la reconceptualización de la construcción de paz en clave crítica de derechos humanos, particularmente en sociedades con conflictos de vieja data como la colombiana, comienza por reconocer el ejercicio de la acción colectiva de las comunidades como una forma real y legítima de ejercer la democracia por fuera de la estructura estatal, pero que bien puede y debe traducir esas demandas o banderas de lucha al lenguaje de las políticas públicas y entrar en el juego burocrático para lograr materializarlas y hacerlas efectivas ante los sujetos políticos que las reclaman.

Tercero, la construcción de paz en clave crítica de derechos humanos solo podrá ser reconceptualizada cuando esos derechos humanos como discurso liberal que Occidente ha enarbolado (con mayor fuerza desde 1948) reconozca los dos elementos anteriores como punto de partida para hacer el viraje de la visión estatalista a la visión verdaderamente relacional de los derechos humanos (Sánchez-Rubio, 2018a). Este reconocimiento de la visión relacional como base de unos derechos humanos no particularistas y con posibilidades emancipatorias permite comprender un concepto de construcción de paz visto desde las necesidades e intereses de las comunidades que han sufrido el conflicto armado 
o que se convierten en víctimas potenciales del mismo como miembros de la población civil.

Las mujeres lideresas que participaron en esta investigación dejaron en evidencia cómo la paz en tanto derecho humano debe reconceptualizarse para que cada vez más pierdan vigencia las posturas que convierten la paz desde un enfoque militarista en el fin último para hacer gala de la soberanía estatal a través del monopolio del uso de la fuerza y la hegemonía del Estado en los distintos territorios que se disputan con grupos armados organizados o delincuenciales.

Es justamente ese enfoque militarista el que se quiso desligar al concepto de derechos humanos y de paz en esta investigación; las mujeres lideresas constructoras de paz participantes dejaron claro que hay "otros mundos posibles" cuando la paz emana desde una perspectiva territorial, local y contextual. Cuando se trabaja por la construcción de paz y por la garantía de los derechos humanos desde el lugar de enunciación y no se pretende imponer una unívoca visión de paz o de derechos humanos por parte de las instituciones estatales y, por el contrario, se propende por otros relatos y discursos que permitan ampliar y nutrir la democracia con el reconocimiento de otros actores, que desde la acción colectiva y la movilización popular pueden dotar de significados otros, a esos conceptos como la paz y los derechos humanos que históricamente han sido serviles al poder hegemónico en contextos locales, nacionales y globales.

Ser mujer en un país como Colombia encarna muchísimos retos en múltiples dimensiones; ahora bien, ser una mujer lideresa, que habita un territorio en conflicto, que ejerce su liderazgo comunitario en esos escenarios y que trabaja de manera incansable por alcanzar la paz, redimensiona los meros retos de la existencia en una sociedad desigual como esta y los sitúa en un contexto de resistencia y de re-existencia.

Ser mujer lideresa en esta convulsionada realidad sociopolítica colombiana es existir, resistir, pero en particular re-existir, pues esto implica que:

(...) los dispositivos de saber, hacer, pensar, sentir y actuar desde las experiencias históricas diferenciadas, para garantizar condiciones de vida dignificadas por el autorreconocimiento, [deben permitir] enfrentar creativamente la desvalorización, el encubrimiento y el silenciamiento en procura de posicionar lugares de enunciación políticos, éticos y epistémicos mediante el desenvolvimiento de una "agencia-otra" (Albán y Rosero, 2016, p. 37).

En esa línea, las luchas por la dignidad de las mujeres lideresas no pueden reducirse a la mera existencia, su papel es crucial para la consolidación de una paz sólida, robusta y duradera. La paz se construye desde esos pequeños escenarios invisibles a la institucionalidad y a los medios de comunicación; la praxis de la 
liberación comienza en la propia corporalidad de estas mujeres como primer territorio de pacificación, y se extiende a los escenarios familiares, sociales y comunitarios como otros territorios de construcción de paces.

En efecto la paz (en su sentido liberal), se nos ha hecho esquiva durante décadas, pero está claro que esa paz completa no es el Acuerdo Final entre Estado y las extintas FARC, ni es la desmovilización o el sometimiento de miembros de grupos armados organizados. La paz también se expresa en esas pequeñas manifestaciones cotidianas de escuchar al otro y de generar empatía. La paz es un llamado a la acción más que el resultado de una negociación. Ser mujer lideresa constructora de paz en Medellín significa anteponer los intereses propios y priorizar los colectivos, porque ese bien común que es la paz no puede en ninguna circunstancia poner en peligro a aquellas mujeres que están convencidas de que otro mundo, mejor por supuesto, es posible. Entonces, vale la pena concluir con una idea que irradió esta investigación: jla paz sin las mujeres no va!

\section{Referencias}

Acosta-Navas, J. P. (2019). Derechos humanos de los pueblos indígenas en clave de pluralismo jurídico e interculturalidad. Anfora, 26(47), 37-60. https://doi.org/10.30854/anf.v26.n47.2019.632

Albán, A.; Rosero, J. (2016). Colonialidad de la naturaleza: ¿imposición tecnológica y usurpación epistémica? Interculturalidad, desarrollo y re-existencia. Nómadas, 45, 27-41. http://www.redalyc.org/articulo. oa? id=105149483004

Ballakrishnen, S. S. (2019). Review of Gender, Alterity and Human Rights, by Ratna Kapur. Irvine School of Law Research Paper, 3, 1-6. https://ssrn.com/abstract=3353543

Centro Nacional de Memoria Histórica, Corporación Región; Ministerio del Interior, Alcaldía de Medellín, Universidad EAFIT; Universidad de Antioquia. (2017). Medellín: memorias de una guerra urbana. https://acimedellin.org/wp-content/uploads/2017/06/medellinmemorias-de-una-guerra-urbana-baja.pdf 
Centro Nacional de Memoria Histórica, CNMH. (2018). Balance del Conflicto Armado. http://centrodememoriahistorica.gov.co/observatorio/wpcontent/uploads/2018/08/General_15-09-18.pdf

Dietrich, W. (2006). Una llamada a muchas paces. En W. Dietrich (Ed.). Schluesseltexte der Friedenforschung (pp. 435-455). https://www.friedensburg.at/uploads/files/wp7_97.pdf

Díez, M. E.; Mirón, M. D. (2004). Una Paz Femenina. En B. Molina Rueda \& F. A. Muñoz Muñoz (Eds.). Manual de Paz y Conflictos (pp. 67-94). Universidad de Granada.

Fernández-Matos, D. C. (2019). La paz sin las mujeres ¡No va! El proceso de paz colombiano desde la perspectiva de género. CIDOB d'Afers Internacionals, 121, 113-133. https://www.cidob.org/es/articulos/revista_cidob_d_ afers_internacionals/121/la_paz_sin_las_mujeres_no_va_el_proceso_ de_paz_colombiano_desde_la_perspectiva_de_genero

Galeano, E. (2015). Luchando por un mundo mejor. Cambio 16, 2217. https://www.cambio16.com/reportajes_data/heroes/esp/

Galeano-Marín, M. E. (2011). Diseño de proyectos en la investigación cualitativa. Universidad EAFIT.

Galtung, J. (2016). La violencia: cultural, estructural y directa. Cuadernos de Estrategia, 183, 147-168. https://dialnet.unirioja.es/servlet/ articulo? codigo $=5832797$

Garrido, A. (2019). Narrativas de resistencia: análisis de tres organizaciones de mujeres en Colombia. Política y Sociedad, 56(1), 107-126. https://doi.org/10.5209/poso.60794

Granada, J. (2019). Un acercamiento conceptual a la definición del líder social. Alma Mater, 9, 1-11.

Instituto de Estudios para el Desarrollo y la Paz, Indepaz. (2020). 1.00o líderes y defensores de DDHH asesinados. 1.000 líderes y defensores de DDHH asesinados. http://www.indepaz.org.co/1-0oo-lideres-y-defensores-de-ddhh/ 
Kapur, R. (2018). Gender, Alterity and Human Rights: Freedom in a Fishbowl. Edward Elgar.

Lederach, J. P. (2008). La imaginación moral. El arte y el alma de la construcción de la paz. Norma.

Mujeres por la Paz (2012). La paz sin las mujeres ;No va! Manifiesto de Mujeres por la Paz. Manifiesto. https://www.rutapacifica.org.co/agenda-de-paz/178la-paz-sin-las-mujeres-no-va-manifiesto-de-mujeres-por-la-paz

Organización de Naciones Unidas, Consejo de Seguridad (2000). Resolución 1325 (2000) Aprobada por el Consejo de Seguridad en su sesión 4213a, celebrada el 31 de octubre de 2000 (Vol. 1325). http://undocs.org/es/S/RES/1325(2000)

Rendón, O. P. (27 de mayo, 2019). La guerra sigue en Antioquia, dice la Comisión de la Verdad. El Colombiano, p. 1. https://www.elcolombiano.com/ colombia/paz-y-derechos-humanos/la-guerra-sigue-en-antioquia-dicela-comision-de-la-verdad-DK 10833606

Restrepo, M. H. (2016). Paz que humaniza, fusiles que destruyen. En E. Rueda, S. V. Alvarado \& P. Gentili (Eds.), Paz en Colombia: perspectivas, desafíos, opciones (pp. 55-60). CLACSO. http://biblioteca.clacso.edu.ar/clacso/ se/20160930124934/Paz_en_Colombia.pdf

Sánchez-Díaz, I. (2017). Mujeres por la paz. Metodologías noviolentas en movimientos pacifistas de mujeres: estudios de casos. Revista de Paz y Conflictos, 1O(2), 265-282. https://revistaseug.ugr.es/index.php/revpaz/ article/view/6477

Sánchez-Rubio, D. (2018a). Derechos Humanos instituyentes, pensamiento crítico y praxis de liberación. Akal.

Sánchez-Rubio, D. (2018b). Seminario: Teorías Críticas de los Derechos Humanos. Conferencia en la Maestría en Derecho de la Universidad de Antioquia.

Segato, R. L. (2013). Las nuevas formas de la guerra y el cuerpo de las mujeres. Pez en el Árbol. 
Villabella, C. M. (2015). Los métodos en la investigación jurídica. Algunas precisiones. Cuestiones constitucionales, Revista mexicana de derecho constitucional, 921-953. https://archivos.juridicas.unam.mx/www/bjv/ libros/8/3983/46.pdf

Wilches, I. (2010). Paz con género femenino. Mujeres y construcción de paz. Fondo de Desarrollo de las Naciones Unidas para la Mujer, UNIFEM. https:// repositorio.unal.edu.co/handle/unal/57178 